\title{
LONGITUDINAL EMITTANCE COMPENSATION IN A PHOTOCATHODE RF GUN INJECTOR
}

\author{
X.J. Wang and I. Ben-Zvi, BrookhaveAccelerator Test Facility, BNL, Upton, NY 11973
}

\begin{abstract}
Electron beam bunch compression directly from photocathode RF gun injector was experimentally observed at the Brookhaven Accelerator Test Facility (ATF). The analysis is presented in this report shows that, the configuration of transverse space-charge emittance compensation photoinjecotor can also be operated in bunch length compression mode for modest amount charge $(<1.0 \mathrm{nC})$, i.e., longitudinal emittance compensation. For a constant laser energy, the electron beam bunch length almost linearly decrease with the RF gun phase, and the compression ratio as large as factor of 30 was experimentally observed for a $40 \mathrm{pC}$ charge. We also discuss the effect of electron beam bunching inside the RF gun on the transverse emittance, and compared with experimental results.
\end{abstract}

\section{INTRODUCTION}

In the past few years, there is tremoundous interest in ualtra -short electron beam production for high energy linear collider, free electron laser, laser accelerators and many other applications [1-4]. Sub-picosecond electron beam can be generated either using the femntosecond laser or magnetic bunch compression, but they are either limited by the total charge or the emittance growth. In this report, we further elucidate a technique using 10 picosecond laser driven photocathode RF gun injector for subpicosecond electron beam generation.

The electron beam micro-bunching in the photocathode RF gun injector was experimental observed at the Brookhaven Accelerator Test Facility (ATF) [5]. The ATF photocathode RF gun injector was designed for transverse space-charge emittance compensation[8]. It consists of a 1.6 cell RF gun mounted on the emittance compensation solenoid magnet, followed by a drift distance and two sections of SLAC type traveling wave linac. The photocathode RF gun injector is driven by a $10 \pm 2$ ps (FWHM) frequency quadrupoled Nd: Yag laser system. We will show in the following sections, that the photocathode RF gun injector designed for the space-charge emittance compensation can be very efficient operated as buncher for sub-picosecond electron beam generation. We will present latest experimetal results, and discuss the effect of electron beam bunching inside the RF gun on the transverse emittance, and compared with experimental results.

\section{LONGITUDINAL EMITTANCE COMPENSATION}

Fig. 1 is the schematic of the layout of space-charge emittance compensation photocathode RF gun injector. Though several authors $[1,6,7,9]$ studied electron beam bunching process inside the RF gun, the roles of the solenoid magnet, drift distance and linac were not discussed. When the space-charge emittance compensation photocathode RF gun injector operated in the small launch phases, the electron beam bunch length can be significantly shorter than the driving laser pulse length (for modest amount charge). The electron beam bunch lenghth compression process can be divided into four stages:

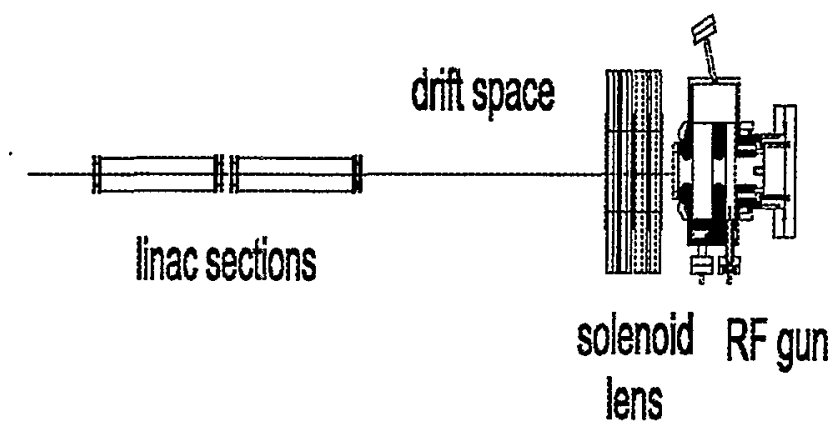

Fig.1 Schematic layout of emittance compensation injector.

Initial launching and expansion: For a $\tau$ ps long laser pulse, the initial electron beam pulse length is much shorter than $\tau$ ps for the initial $\tau$ ps time interval because the electrons come out photocathode are nonrelativistic. For example, for a peak field of 100 $\mathrm{MV} / \mathrm{m}$ on the cathode, the electron beam bunch length after initial laser pulse during tion is about one sixth of the laser pulse length.

After the inital stage, the electron beam will expand because the head of the bunch moving faster than the tail becaused it gains more energy. This process usually last several laser pulse duration.

$R F$ compression inside the $R F$ gun cavity: For electron beam launches in small RF phase, the tail of the beam gains energy faster than the head. There are several paper discuss the bunch lenght compression inside the RF gun $[1,6,7,9]$ cuased by the RF force. For normalize field $\alpha=\mathrm{eE} / \mathrm{mc}^{2} \mathrm{k}$ mage between 1 and 4 , reference 9 gives a analytical expression for ratio of

*Work supported by the Department of Energy, contracts DE-AC03-76SF00515 and DE-AC02-76CH00016 
electron beam bunch length change $\Delta l$ to the laser pulse lenghth $\mathrm{I}_{\text {laser }}$

$$
\begin{aligned}
& \frac{\Delta l}{l_{\text {laser }}}=\frac{1}{\sqrt{\alpha^{* 2}}\left(1+\cos \left(\phi_{0}\right)\right)^{2}+1} \\
& \frac{2 \alpha\left(1-\sin \left(\phi_{0}\right)\right) \cos \left(\phi_{0}\right)}{1+2 \phi_{0} / \pi}
\end{aligned}
$$

where $\alpha^{*}$ is so called modified normalized field, $\phi_{0}$ is the electron beam launch phase, $\alpha^{*}$ can be writtern as,

$$
\alpha^{*}=\frac{\alpha+\frac{\alpha^{2}}{6} \sqrt{\sin \left(\phi_{0}\right)}}{1+\frac{\sqrt{\sin \left(\phi_{0}\right)}}{6}}
$$

Fig.2 shows that the bunch lenght of the electron beam is almost linearly decrease with the electron beam launch phase[6]. The relative energy spread of the beam also almost linearly increase with the decreasing phasing after the launching phase below the phase $\phi_{0}$ which corresponding to the electron beam exit phase $\pi / 2$.

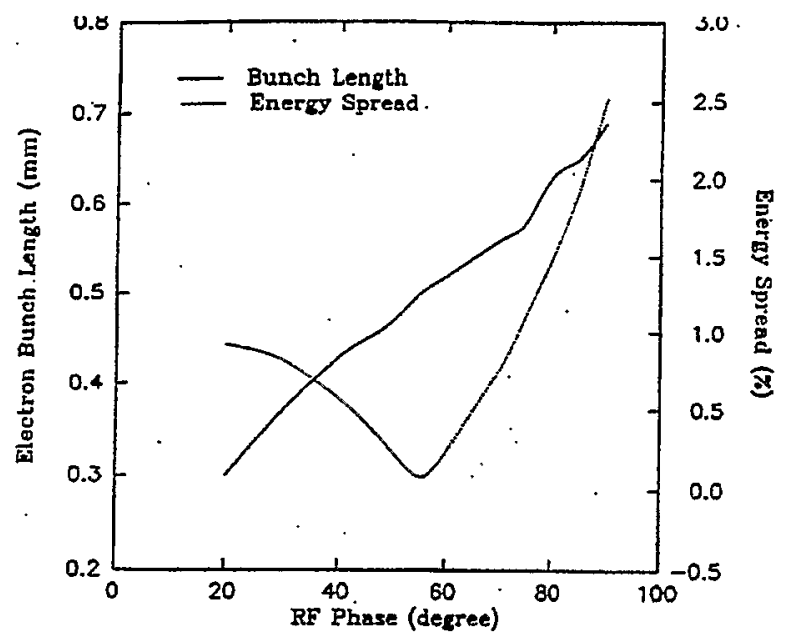

Fig.2 Electron beam bunch lenghth and energy spread as function of the launching phase for a $2.5 \mathrm{ps}$ laser pulse.

Drift space bunch compression: Solenoid magnet plays an important role in transverse phase realignment in space charge emittance compensation photocathode RF gun injector, and hence achieaving the emittance compensation. If the injector to be operated as a bunch compressor, solenoid magnet is critical in preserving and further compress the electron beam to achieave subpicosecon bunch length. The electron beam bunch lenght change in the drift space can be express as,

$$
\Delta l=\int \frac{1}{2}\left(x^{2}+y^{, 2}\right)+\left(L-L_{0}\right)\left(1-\frac{\delta}{\gamma^{2}}\right)-L \frac{\delta}{\gamma^{2}}
$$

The first term represent bunch lengthening. For a $1 \mathrm{~mm}$, radius laser spot, the beam divergence $x^{\prime}$ at gun exit is about $10 \mathrm{mrad}$. After one meter drift distance (roughly the drift distance between the gun and linac), bunch lengthed about $0.3 \mathrm{ps}$ due to the beam divergence. The focusing of the solenoid magnet reduce the beam diverence about a factor of 3 to 5 , this reduce the bunch lengthening caused by the beam divergence to less than $30 \mathrm{fs}$. The second term of Eq. 3 is negligible. For a 10 ps laser pulse, the energy spread of the electron beam is about 5 percent when beam was launched in the small phase, this will leads to about $1.5 \mathrm{ps}$ bunch lenght reduction in the drift space. For a 10 ps laser pulse, electron bunch length can be easily reduce by factor of 3 to 4 inside the gun (if psace charge effect is small), so the bunch lenght of the e-beam at gun exit is about 3 to 2 ps, and after further bunch compression in the drift distance, sub-picosecond electron beam can be generated with modest charge. Our experiment confirmed above analysis [5]. We have experimentally measured $370 \mathrm{fs}$ (FW) electron beam with $40 \mathrm{pC}$ charge.

Longitudinal emittance compensation through linac: The linac plays very similar role for longitudinal emittance compensation as for transverse space-charge emittance compensation. It reduces space charge effects and preserve the electron beam bunch lenghth. As electron beam is accelerated through linac, followings are realized,

1. Further bunch length reduction on the order of 5 to $10 \%$ caused by the early injection due to the relative low energy from the RF gun $(\gamma<10)$.

2. Space charge reduction as $1 / \gamma^{2}$.

3. Energy spread will be reduction as $1 / \gamma$, and the final energy spread will be determined by the bunch lenghth $\left(\Delta \mathrm{l}^{2} / 8\right)$ and the wake field.

Summarize 1 to 3 , after linac acceleration, the short bunch lenghth was preserved, and achieve smaller energy spread (assume will be dominated by the bunch effect), hence longitudinal emittance compensation.

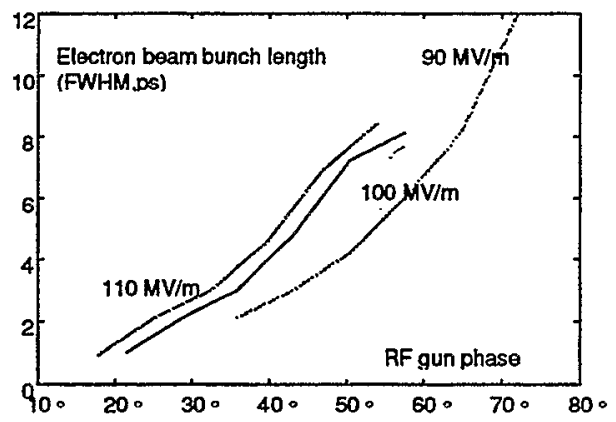

Fig. 3 Electron beam bunch length as function of the RF gun phase for 3 electric fields. 
The electron beam bunch lenghth was measured for three electric fields at different RFphases(Fig.3).It confirmed basic features predicted in our discussion. We should pointe out that,the experiments was carried out for a constant laser energy, and it seems no space-effect was observed as bunch was compressed. This can be explained by the Schottky effect.

\section{DISCUSSION}

Just as any bunch compressor, there are many debunching effects in a photocathode RF gun injector. Directly borrow from the analysis from reference 1 , the bunchlenghthening caused by the beam emittance inside the $\mathrm{RF}$ gun can be reduced much less than $100 \mathrm{fs}$. Space charge effect will limit the photocathode RF gun injector operating as a bunch compressor in modest charge $(<1.0$ nC). We have observed recently significant bunch comrpression for a charge of $0.7 \mathrm{nC}$ for a peak current of $160 \mathrm{~A}$ (Fig.4).

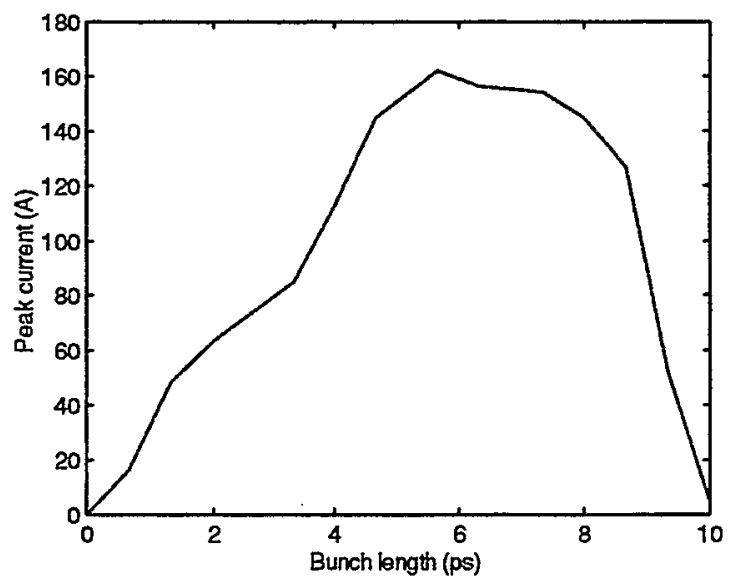

Fig.4 Electron beam charge distribution for $0.7 \mathrm{nC}$ charge, bunch compression is about a facot of 2.5 comparing the laser pulse.

Since the most applications require not only short bunch, but also the high-brightness. The emittance of the compressed beam as the function of the RF gun phase were measured for two RF injectos [5,11]. The result is reproduced in Fig. 5 [11]. One of the most interesting feature is the nonlinear emittance decrease with the RF gun phase. The measurements were performed with a constant laser energy, so the charge in the beam decrease almost linearly with the RF gun phase due to Schottky effect. To the first order, the space charge effect remains roughly constant. Eletron beam produced by the RF gun acquires most its transverse momentum, hence its $R F$ emittance at the exit of the RF gun [10]. As electron beam bunch lenghth was compressed inside the gun, the quadratic dependency of the RF emittance on the bunch length qualitatively agrees with the measurement. Calculated RF emittance [12] using our beam parameters is about one order magnitude smaller than the measured value. This means meausred emittance was dominated by the space charge effect, which we pointed out earlier should remain roughly constant. The physics lies in the correlation between the RF emiftance and space induced emittance [12]. It is well known that space charge emittance growth will be minimized for smaller beam in the transport line. Silimar argument applied for space charge emittance growth inside the RF gun.

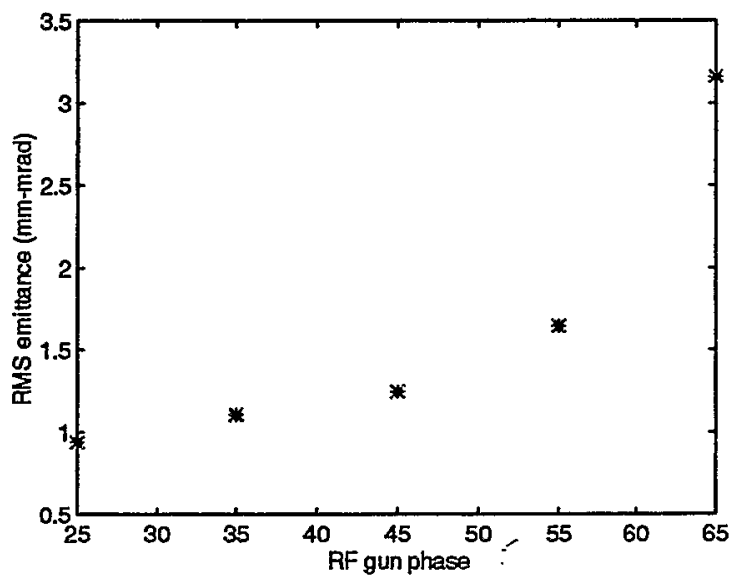

Fig.5 Normalize RMS emittance as function of the the RF gun phase for a constant laser energy.

\section{ACKNOWLEDGEMENT}

Authors gratefully acknowledge discussion with D.T. Palmer, the technical support form Mr. W. Cahill, R. Harington and M. Montemgo made the work present here possible. This work is supported by the U.S. D.O.E. contract No. DE-AC02-76CH00016.

\section{REFERENCES}

[1] L. Serafini, R. Zhang and C. Pellegrini, Nucl. Instr. and Meth. A387 (1997) 305 - 314.

[2] P. Kung, H. Lihn and H. Wiedemann, Phys. Rev. Lett. 73, 967 (1994).

[3] B.E. Carsten and S.J. Russell, Phys. Rev. E. 53, R2072 (1996).

[4] J.B. Rosenzweig, N. Barov and E. Colby, IEEE Trans. on Plasma Sci. Vol.24, No.2, 400 (1996).

[5] X.J. Wang, X. Qiu and I. Ben-Zvi, Phys. Rev.E. 54, R3121 (1996)

[6] X.J. Wang, UCLA P.h.D thesis (1992).

[7] L. Serafini, IEEE Trans. on Plasma Sci. Vol.24, No. 2, 421 (1996).

[8] B.E. Carlsten, Nucl. Inst. and Meth. A 285 (1989) 313.

[9] B.E. Carslten, D.C. Nguyen and R. L. Sheffeld, LA-UR-96-424 (1996).

[10] K.J. Kim, Nucl. Inst. and Meth. A 275 (1989) 201.

[11] D.T. Palmer, X.J. Wang et al, to be published in the Proc. of the $7^{\text {th }}$ Advanced Accelerator Concepts Workshop, BNL-63808 (1996).

[12] C. Travier, Nucl. Inst. and Meth. A 340 (1994) 26. 